\title{
Hard x-ray lasers take their first steps towards nanostructure solution
}

\author{
Single mimivirus particles intercepted and imaged with an X-ray laser \\ Authors: M. Marvin Seibert, et al. \\ Nature 470, 78-81 (2011) doi:10.1038/nature09748
}

Femtosecond X-ray protein nanocrystallography

Authors: Henry N. Chapman, et al.

Nature 470, 73-77 (2011) doi:10.1038/nature09750

Recommended with a commentary by Simon J. L. Billinge, Department of Applied Physics and Applied Mathematics, Columbia University, and Condensed Matter Physics and Materials Science Department, Brookhaven National Laboratory:

Two recent, back to back [1,2], landmark publications in Nature mark the first steps taken by hard x-ray free electron lasers (XFELs) in nanostructure determination. The XFEL is the Linear Coherent Light Source (LCLS) at the Stanford Linear Accelerator Center (SLAC) in California. It is a linear accelerator (linac) based x-ray source which is a significant departure from the now familiar synchrotron storage ring sources. Synchrotrons have had, and continue to have, enormous scientific impact providing intense beams of highly coherent hard and soft $\mathrm{x}$-rays to materials scientists. What are the advantages of a linac based system and what science can be done there that is not possible at a synchrotron?

Both types of sources produce short, intense, pulses of x-rays with high coherence, somewhat like a pulsed optical laser. In the synchrotron, pulses of electrons circulate in a ring close to the speed of light. The x-ray pulse duration depends on the length of the electron bunch and is typically 10-100 picosecond duration. The number of x-rays in the pulse is $\sim 10^{10}$. The linac based source dumps the entire energy of the beam after each pulse, which makes it expensive to run, but the removal of the need to maintain stable, long-lived bunches in the ring (for days and weeks at a time) mean that much shorter and more intense pulses are possible. For LCLS, the numbers are more like $10^{12}-10^{13}$ photons per 10 fs pulse.

X-ray diffraction experiments suffer from the parsimonious Thomson scattering cross-section, $7 \times 10^{-25} \mathrm{~cm}^{2}$ : the probability of an x-ray getting scattered is very small. X-ray diffraction works as a viable technique because we put bulk 
samples with a large number $\left(\sim 10^{20}\right)$ of atoms in the beam, which has sufficient photons ( $10^{10}$ per second $)$, and we can measure for sufficiently long, to get good statistics $\left(10^{4}\right)$ in the scattered x-ray signal. But what if we want to measure a single nanoparticle consisting of just $10^{2}-10^{3}$ atoms? We need to impinge $\gg 10^{20} \mathrm{x}$-rays on the sample.

This is not possible with current synchrotron sources, making nanostructure determination one of the grandest of our grand challenges in science [3]. Nanostructures can be solved from multiple copies of the nanoparticle in special cases; from a powder [4] or from a single crystal of aligned nanoparticles [5]. But what about single nanocrystallography, the solution of the atomic structure from a single nanoparticle? If we could mount and rotate the nanoparticle, and irradiate it for a long enough time, as is done with (much larger) single crystals now at synchrotrons and on lab machines, we could amass enough statistics to get $\mathrm{x}$-ray intensities that can be inverted to get the desired 3D structure. Notwithstanding the outrageous technical challenges of doing this, it is not possible even in theory because of beam damage. A beam that could put enough photons on the sample in each pulse to make this feasible in a reasonable time produces beam damage and the nanoparticle doesn't survive long enough to amass the statistics. The XFEL beams are so intense they explode the nanoparticle in a single shot, but they are sufficiently short in duration that the end of the pulse leaves the exploding nanoparticle before the nanoparticle has blown up, taking a diffraction pattern of the undamaged particle to the detector even as it detonates in the wake of the departing x-ray pulse. At least that's the theory. The first real demonstrations of this are now in and reported in [1] and [2] and it works in practice. These are baby steps towards the goal of single nanocrystallography, but the results look promising and impressive. In [1] an image of a non-crystalline object, in this case a large $100 \mathrm{~nm}$ diameter virus, has been obtained with a $10 \mathrm{~nm}$ resolution. In [2] a structure solution of a protein has been obtained from a nanometer sized crystal of nanoparticles with $\sim 0.9 \mathrm{~nm}$ resolution. The real-space resolution will naturally go up in the future as the $\mathrm{x}$-ray energy of the beam is increased and the resulting x-rays have a shorter wavelength, a planned and straightforward technical development. We are still some way away from single nanocrystallography. The single nano-object that has been imaged [1] (no complete 3D reconstruction has been done) is large at $100 \mathrm{~nm}$ and imaged at $10 \mathrm{~nm}$ resolution, still far from atomic resolution. Close to atomic resolution has been achieved in the nano-sized crystals of proteins, but these are not 3D structures of the individual proteins, it is protein crystallography on nano-sized protein crystals. It is still a huge accomplishment and should have significant scientific impact. When we say that a protein "doesn't crystallize" we mean that it doesn't grow into large, micron sized, crystals with good structural coherence (and therefore good diffraction qualities in the beam). We don't know how many of the "poorly crystallizing" proteins will have nanocrystals floating around in the crystallization vial that can be solved in the XFEL, but a good guess would be "quite a few". This is important since, despite the 
almost industrial proportions of protein crystallography efforts at synchrotrons these days, the proportion of all proteins that have good crystallization properties remains small.

Nanocrystallography at the XFEL is a big operation. Each of the two initial papers has $>80$ authors, exemplifying the huge teams that were put together to solve the myriad problems of bringing this work to fruition, moving materials science and protein crystallography in the same direction of high energy physics with large collaborations needed to make the big breakthroughs. In this case, future publications are likely to have small teams use the technologies built by the big teams and it will be small group science again. But as the capabilities develop at the XFELs, demand for time will become intense. At LCLS there is one linac serving 6 endstations, only one of which can take beam at any given time. Other developments to keep an eye on in the future are hybrid machines such as energy recovery linacs (which combine linacs with curved sections) and ultimate storage rings. These are at an early research stage, for example prototype hardware being developed and tested at Cornell university, but they can combine some of the best attributes of the FELs and the synchrotrons.

\section{References}

[1] M. M. Seibert et al., Nature 470, 78 (2011).

[2] H. N. Chapman et al., Nature 470, 73 (2011).

[3] S. J. L. Billinge and I. Levin, Science 316, 561 (2007).

[4] P. Juhás, D. M. Cherba, P. M. Duxbury, W. F. Punch, and S. J. L. Billinge, Nature 440, 655 (2006).

[5] P. D. Jadzinsky, G. Calero, C. J. Ackerson, D. A. Bushnell, and R. D. Kornberg, Science 318, 430 (2007). 